\title{
Repercusiones de la roncopatía y respiración bucal en el crecimiento facial
}

\author{
Impact of snoring and mouth breathing on facial growth
}

\author{
Alfredo Santamaría C¹, Felipe Fredes C¹.
}

\begin{abstract}
RESUMEN
Los trastornos respiratorios obstructivos del sueño corresponden a un amplio espectro de patologías que incluyen a los roncadores primarios, el síndrome de resistencia de vía aérea superior y al síndrome de apnea obstructiva del sueño (SAOS). Dentro de sus manifestaciones clínicas se encuentra la roncopatía y la respiración bucal, las cuales generan alteraciones en el crecimiento craneofacial del niño pudiendo ser la causa de un SAOS residual. El objetivo de esta revisión es analizar las alteraciones del crecimiento craneofacial en niños generadas por la roncopatía y respiración bucal.

Palabras clave: Roncopatía, respiración bucal, crecimiento craneofacial.
\end{abstract}

\begin{abstract}
Obstructive sleep breathing disorders correspond to a broad spectrum of diseases that include primary snorers, upper airway resistance syndrome and obstructive sleep apnea syndrome (OSAS). Its clinical manifestations include snoring and mouth breathing, which generates alterations in the craniofacial growth of the child that may be the cause of a residual OSAS. The objective of this review is to analyze the alterations of craniofacial growth generated by snoring and mouth breathing in children.
\end{abstract}

Key words: Snoring, mouth breathing, craniofacial growth.

\section{INTRODUCCIÓN}

Los trastornos respiratorios obstructivos del sueño corresponden a un amplio espectro de patologías que incluyen a los roncadores primarios, el síndrome de resistencia de vía aérea superior y al síndrome de apnea obstructiva del sueño ${ }^{1}$. Estas patologías tienden a ser continuas y progresivas en su evolución, y se presentan durante el sueño.

\footnotetext{
1 Médico del Servicio de Otorrinolaringología, Hospital Guillermo Grant Benavente, Concepción.
} 
La prevalencia varía según el tipo de trastorno, siendo la prevalencia de los roncadores primarios de $2,4 \%$ al $34,5 \%{ }^{2}$ versus 1,2 a $5,7 \%$ en el síndrome de apnea obstructiva del sueño ${ }^{3}$. En Chile, el año 2012 se determinó una prevalencia de roncadores primarios en población pediátrica de $18 \%{ }^{4}$, en sectores de bajos ingresos.

Factores relevantes como causas de estas patologías en el niño son: la hiperplasia adenoamigdalina, la obesidad y las enfermedades neuromusculares $y / 0$ malformaciones craneofaciales ${ }^{1}$. El gold standard para su diagnóstico es la polisomnografía ${ }^{3}$, examen caro, difícil de realizar en niños y poco disponible en nuestro medio. Como alternativas de estudio, la Academia Americana de Pediatría, recomienda la realización de oximetría nocturna, grabaciones nocturnas del sueño o polisomnografía o poligrafía ambulatoria ${ }^{3}$.

La presentación clínica se manifiesta como: síntomas diurnos, nocturnos y hallazgos al examen físico ${ }^{1,3}$. En cuanto a los síntomas diurnos que se pueden presentar tenemos: bajo rendimiento escolar, hiperactividad, agresividad, somnolencia, trastornos del aprendizaje y cefalea matinal ${ }^{5}$. Los síntomas nocturnos son los más conocidos y son los que motivan a los padres a consultar y consisten en ronquidos, apneas, movimiento corporales frecuentes, respiración dificultosa, respiración paradojal, enuresis y sueño no reparador. Al examen físico se puede encontrar: respiración bucal, hiperplasia amigdalina, obesidad, micrognatia/retrognatia, paladar alto y estrecho, con o sin apiñamiento dentario anterior, macroglosia, retraso pondoestatural y alteraciones del desarrollo normal craneofacial, siendo estas últimas no siempre consideradas y el motivo de esta revisión.

\section{CRECIMIENTO CRANEOFACIAL NORMAL}

La cefalometría consiste en el estudio y medición de la cabeza humana. Para realizar estas mediciones se utilizan diversos puntos de referencia detallados en la Figura 1.

En los estudios analizados en esta revisión se utilizó principalmente la cefalometría de Steiner ${ }^{6}$, por lo cual, a modo de introducción a la cefalo-

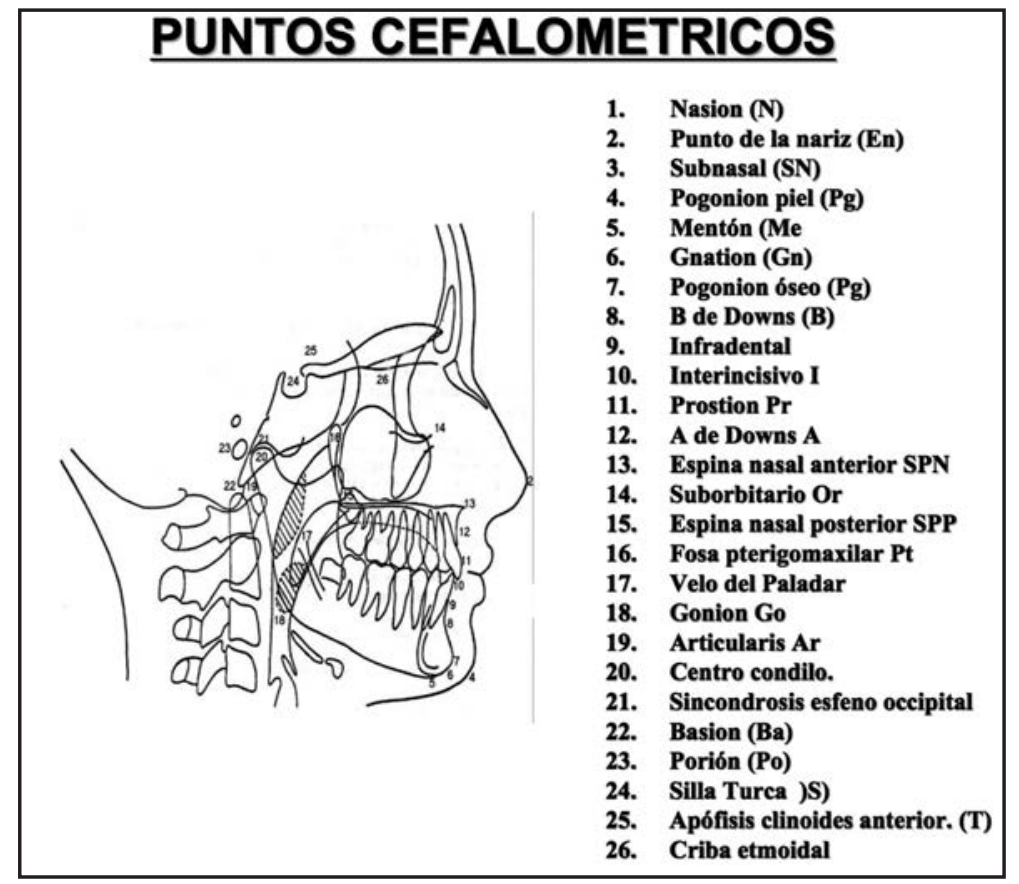

Figura 1. Puntos cefalométricos. 


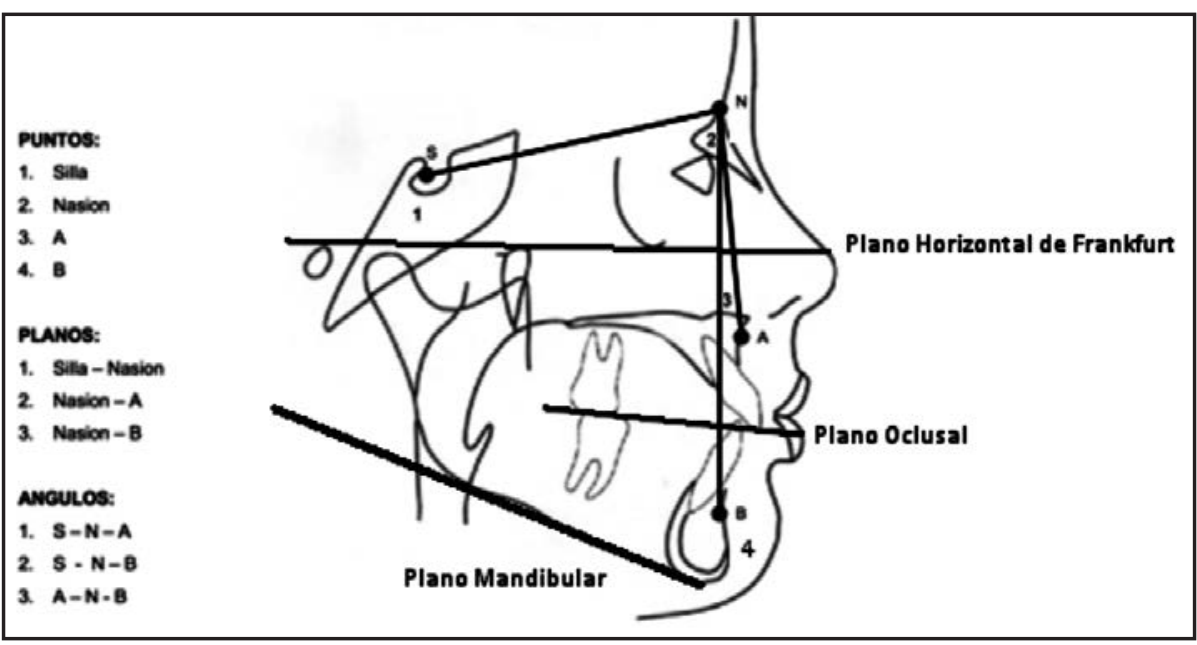

Figura 2. Puntos, planos y ángulos cefalométricos de Steiner.

metría, se detallarán los puntos, planos y ángulos utilizados en esta revisión (Figura 2).

- Silla (S): centro de la silla turca del esfenoides.

- Nasion (N): unión de la sutura frontonasal con los huesos propios de la nariz.

- A: Punto subespinal se localiza en la concavidad anterior del perfil óseo del maxilar entre la espina nasal anterior y el reborde alveolar.

- B: Punto supramental, se localiza más posterior de la concavidad anterior en el perfil óseo de

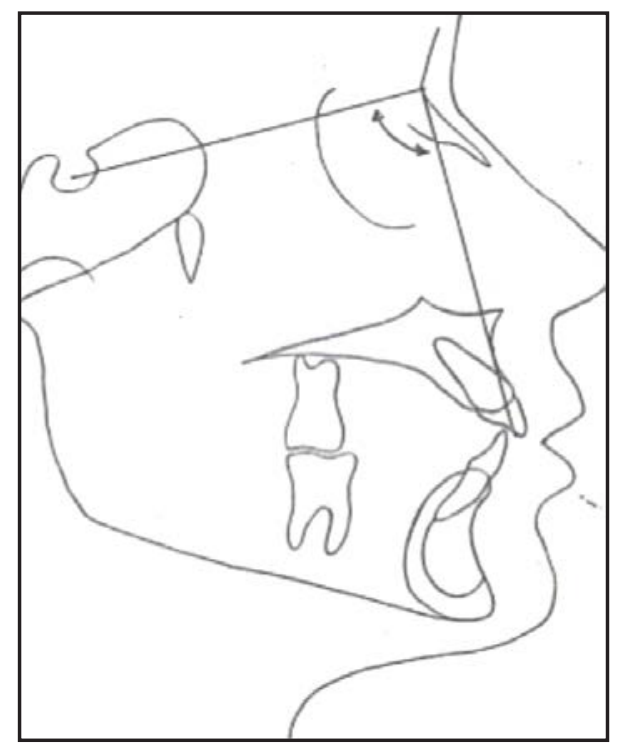

Figura 3. Ángulo SNA. la mandíbula ubicado entre el Pogonion y el reborde alveolar.

- Plano Silla-Nasion (S-N): Plano que se extiende desde el centro de la silla turca del esfenoides hasta la unión de la sutura frontonasal con los huesos propios de la nariz.

- Plano Nasion - A: Plano que se extiende desde el Nasion hasta el punto A o subespinal.

- Plano Nasion - B: Plano que se extiende desde el Nasion hasta el punto B o supraespinal.

- Ángulo Silla - Nasion-A (SNA): ángulo conformado entre planos S-N y N-A.

- Ángulo Silla - Nasion - B (SNB): Ángulo conformado entre los planos S-N y N-B.

El desarrollo normal de la cara se caracteriza por el crecimiento de los maxilares hacia abajo y hacia delante. Esta dirección de crecimiento da como resultado un patrón morfológico facial normal y estable. Durante el crecimiento fisiológico de la cara las lentas variaciones de la morfología que puedan ocurrir van encaminadas a mantener el patrón facial en equilibrio. La cara tiende a la anterrotación, esto significa que los planos mandibular y oclusal se van cerrando respecto al plano Silla-Nasion (SN) ${ }^{7}$.

Entre los 5 y 17 años, el ángulo conformado por el plano Silla-Nasion y el plano Nasion-punto A 0 Subespinal conocido como SNA (Figura 3), aumenta 2 grados y el ángulo conformado entre el plano Silla-Nasion y el plano Nasio-punto B o Supramental 


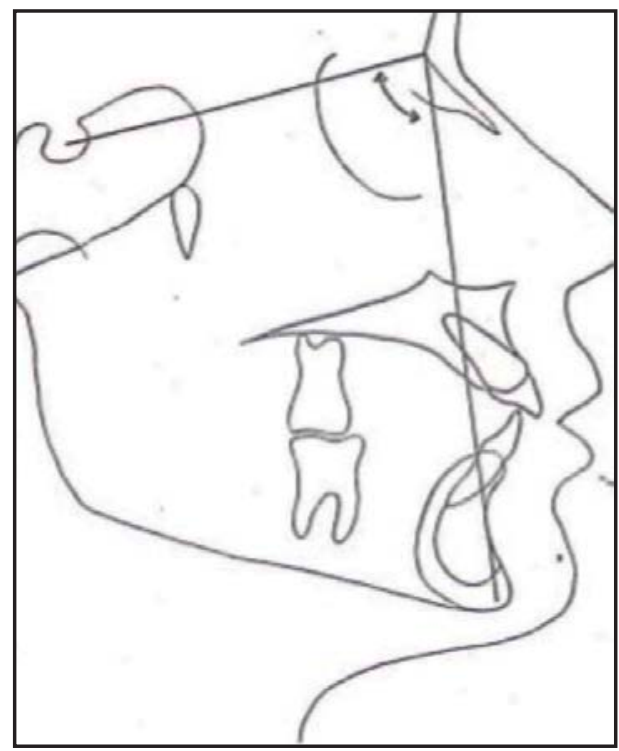

Figura 4. Ángulo SNB.

conocido como SNB (Figura 4) aumenta 4 grados . Por otro lado, el ángulo del plano mandibular, formado por la tangente a la mandíbula y el plano horizontal de Frankfurt disminuye en 8 grados. Sin embargo, el ángulo NSGn (ángulo entre plano SN y tangente que pasa por punto Gnation, el cual corresponde al punto de intersección entre plano mandibular y plano facial de Downs, plano que va desde el Nasion hasta el Pogonion) que determina el eje facial, se mantiene y anterrota en 1 grado (Figura 5).

Este patrón de crecimiento normal será estable mientras no cambien las condiciones de forma y función que lo mantienen en equilibrio. Es por esto que el patrón de crecimiento normal se puede alterar a causa de algunos cambios ambientales independientes de la predeteminación genética, por ejemplo, la respiración bucal.

\section{ALTERACIONES CRANEOFACIALES PRODUCIDAS POR LA RESPIRACIÓN BUCAL}

En 1981 Harvold y cols 8 , realizó un experimento con primates para confirmar la relación entre obstrucción nasal, la respiración bucal y las maloclusiones dentales y el desarrollo craneofacial. Para esto utilizó 42 monos Rhesus nacidos en el Centro de Biología

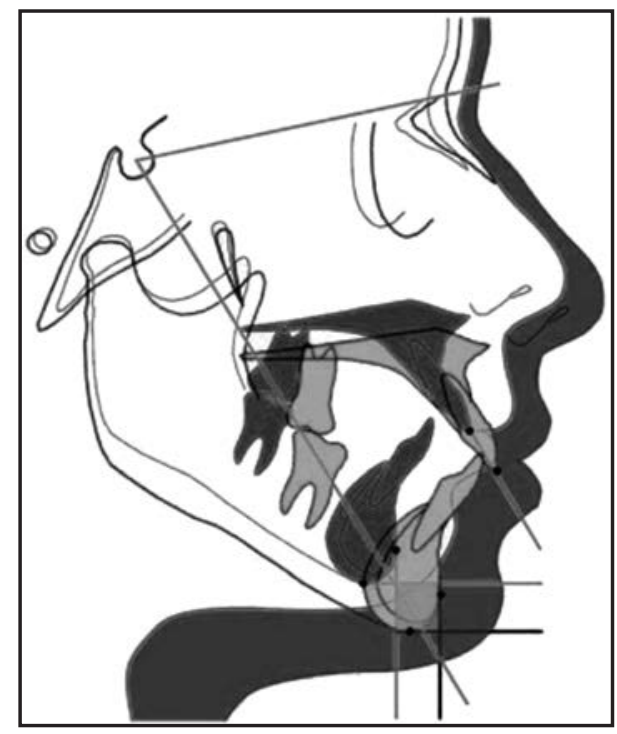

Figura 5. Dirección del crecimiento normal de la cara.

de Primates de la Universidad de California. Los animales fueron distribuidos en parejas del mismo sexo y con el mayor de similitudes de morfología facial, para ser 23 casos y 23 controles pareados. A cada uno de los casos se le bloqueó la cavidad nasal con una válvula de silicona en cada fosa nasal de $1 \mathrm{~cm}$ de largo fijados con una ligadura al septum nasal obstruyendo completamente el paso de aire durante la inspiración. Durante la intervención, que duró 3 años, los animales fueron controlados cada 3 meses realizando cefalometría, fotografía de la cara y dentición, moldes de la dentición y peso. Posterior al retiro de la válvula, fueron evaluados cada 6 meses. Los hallazgos fueron los siguientes:

- Labios: En el grupo experimental todos mantenían los labios abiertos y desarrollaron respiración bucal. Además se observó la tendencia de presentar una escotadura en el labio superior debido a una acción especial de los músculos elevadores del labio. Asociado a esto, la escotadura era ocupada por la lengua protruida. Esta escotadura tendió a la desaparición una vez reiniciada la respiración nasal posintervención.

- Lengua: La lengua de los animales experimentales se observó protruida, más alargada y estrecha con un dorso más delgado dejando 
un espacio entre el paladar y la lengua. Estos cambios también fueron reversibles.

- Dentición: Los hallazgos fueron un estrechamiento del arco mandibular con una disminución del largo del arco maxilar provocando una mordida cruzada.

- Movimientos mandibulares: La respiración bucal indujo mesio-oclusión, protrusión maxilar, mordida abierta y mordida dual. La mesio-oclusión se presenta cuando la cúspide mesio-vestibular del primer molar superior ocluye por delante de la cúspide mesiovestibular del primer molar inferior. Los dientes de la arcada superior y el maxilar superior de sitúan mesializados al maxilar inferior, que se encuentra retraído.

- Cefalometría: Durante la intervención el grupo experimental presentó incrementos estadísticamente significativos en los planos nasion-palatal y nasion-sinfisiario e incremento de los ángulos SN-palatal, SN-borde inferior mandibular y gonial.

- Electromiografía y comportamiento: Los respiradores bucales reclutaron los músculos intrínsecos de la lengua y los genioglosos para la protrusión de la lengua, el elevador del labio incrementó su actividad para mantener la boca abierta, el geniohioideo y los músculos digástricos fueron reclutados para bajar la mandíbula y la porción anterior de los temporales y los pterigoideos laterales fueron usados para avanzar la mandíbula.

Si bien en este trabajo no todos los animales del grupo experimental presentaron los mismos cambios, sí se puede concluir que al presentar una obstrucción nasal todos los respiradores bucales presentaron la tendencia a incrementar el largo de la cara, aumentar el ángulo gonial y un plano mandibular más vertical. Además presentaron contracturas musculares que cambiaron la posición de los labios, lengua y mandíbula y todos los casos desarrollaron diferentes tipos de maloclusión.

En niños con SAOS se han reportado las siguientes características craneofaciales ${ }^{9}$ :

- Base de cráneo anterior acortada

- Ángulo de flexión de la base de cráneo menos obtuso

- Mandíbula retroposicionada
- Mandíbula pequeña

- Maxilar pequeño

- Paladar blando largo

- Disminución del espacio aéreo

- Posición inferior del hioides

- Aumento del largo de la cara

\section{HIPERPLASIA ADENOAMIGDALINA $Y$ CRECIMIENTO CRANEOFACIAL}

Dado que la principal causa de los trastornos respiratorios del sueño (TRS) es la hiperplasia adenoamigdalina, la adenoamigdalectomía ha sido considerada por años como la solución para este problema. Sin embargo, se ha reportado aproximadamente hasta en $20 \%$ de persistencia sintomática en pacientes operados ${ }^{10}$. Debido a lo anterior, se han estudiado factores predictores del fracaso de la cirugía, identificándose 4 principalmente: hipertrofia de cornetes inferiores, desviación nasoseptal, alteraciones morfológicas de la mandíbula y Mallampati 3 ó 411. Lo anterior confirma la importancia de la alteración del desarrollo craneofacial en la resolución de la sintomatología de Ios pacientes con TRS, principalmente del complejo nasomaxilar y de la mandíbula.

El año 2002, Zettergren-Wijk y cols ${ }^{12}$, publicaron su estudio sobre el efecto de la amigdalectomía en cuanto al desarrollo de la dentición y la morfología facial en niños con síndrome de apnea obstructiva del sueño. En este estudio participaron 28 niños, 14 fueron amigdalectomizados y 14 niños sanos en el grupo control, promedio de edad de 5,7 años. Se realizaron evaluaciones ortodóncicas posoperatorias al año y a los 3 años en ambos grupos, con moldes de la mordida, cefalometría y fotos de la cavidad oral. La evaluación preoperatoria demostró alteraciones de la mordida, una disminución del ancho del arco maxilar y un crecimiento más vertical de la cara de los niños con SAOS. En los controles posoperatorios Ios niños con SAOS al recuperar la respiración nasal retomaron el crecimiento mandibular y facial, disminuyendo el crecimiento vertical de cara y mandíbula y aumentando el crecimiento horizontal de cara, maxilar y mandíbula, pero sin llegar a parámetros normales para su edad. En todas las mediciones se produjo un cambio acelerado durante el primer año que disminuyó en los 
años posteriores. Por lo tanto, la conclusión de este estudio fue solucionar el problema respiratorio lo más precoz posible en los niños para retomar el crecimiento normal de la cara.

\section{TRATAMIENTO DEL SAOS RESIDUAL POSADENOAMIGDALECTOMÍA}

Kim y $\operatorname{cols}^{13}$, evaluaron la frecuencia de un paladar elevado y estrecho y/o de una mandíbula retraída en niños con trastornos respiratorios del sueño diagnosticados por polisomnografía. Se evaluaron 400 niños entre 2-17 años, excluyéndose aquellos con obesidad 0 alguna genopatía. En cada uno se evaluó la vía aérea mediante la escala de Mallampati, el tamaño de las amígdalas (1 al 4), características del paladar blando, presencia de paladar duro alto y estrecho, posición de los dientes, evaluación de la oclusión dental, tamaño de los cornetes inferiores, presencia de desviación septal, cefalometría centrada en el largo de la cara y la posición del maxilar y mandíbula y finalmente una polisomnografía. 290 presentaron hiperplasia amigdalina grado 3-4, 385 una mandíbula pequeña y 347 un paladar duro estrecho, demostrando la alta frecuencia de alteraciones del desarrollo craneofacial en niños con SAOS. Del total de 400 niños, 378 fueron sometidos a adenoamigdalectomía y de ellos 167 (42\%) presentaron persistencia de los síntomas con un índice de apnea-hipopnea >2. De estos 167 niños, 136 fueron clasificados como paladar estrecho y 125 con una mandíbula pequeña. Sin embargo, el grupo que respondió a cirugía presentaba alteraciones craneofaciales similares. Este trabajo concluyó que el principal factor predictor de la persistencia de los síntomas es la presencia de un valor de 3 ó 4 en la escala de Mallampati, lo que probablemente está condicionado por la falta de desarrollo de la mandíbula.

La expansión rápida del maxilar (ERM) 0 distracción maxilar se ha planteado como parte importante del tratamiento para la corrección de las alteraciones craneofaciales producidas por la respiración bucal en niños con TRS con sintomatología residual posadenoamigdalectomía. La ERM, se produce aplicando una fuerza lateral contra la dentición posterior del maxilar, produciendo la separación de la sutura media palatina. El aparato utilizado consiste en un tornillo central de expansión de $1,5 \mathrm{~mm}$ de diámetro con 4 brazos anclados en los dientes seleccionados, aplicando una fuerza de hasta $1 \mathrm{~kg}$. La ERM produce un aumento en la dimensión transversa del hueso basal maxilar, logrando la corrección de la mordida cruzada preexistente y aumentando la longitud del arco maxilar $^{14}$ (Figura 6).

Pirelli y cols $^{15}$, en 2004 trataron a un grupo de niños con SAOS con expansión rápida del maxilar. Todos los niños de este estudio fueron evaluados por un ortodoncista y un otorrinolaringólogo. Como criterios de inclusión debían presentar ausencia de hiperplasia adenoamigdalina (22 ya habían sido sometidos a una adenoamigdalectomía) un índice masa corporal $<24 \mathrm{~kg} / \mathrm{m}^{2}$, presencia de una malaoclusión caracterizada por un contracción del maxilar, apiñamiento dentario anterior (candidato a tratamiento con ERM) y un SAOS diagnosticado por polisomnografía. El protocolo de ERM utilizado constó de dos fases, una fase activa de 10-20 días de duración con una expansión de $1 \mathrm{~mm}$ al día, seguida de una fase de retención por 6 a 12 meses de duración. Participaron 31 niños entre 6 y 12 años. Todos los niños presentaban contracción del maxilar resultando en un paladar duro alto y estrecho asociado a malaoclusión caracterizada por mordida cruzada uni o bilateral. A todos los niños se les realizó una polisomnografía previo al inicio del tratamiento, a los 4-6 semanas de tratamiento y 4 meses postérmino de tratamiento. El índice promedio de apnea/hipopnea previo a la intervención fue de 12,18 $\pm 2,6$. Después de 4 meses de terminada la

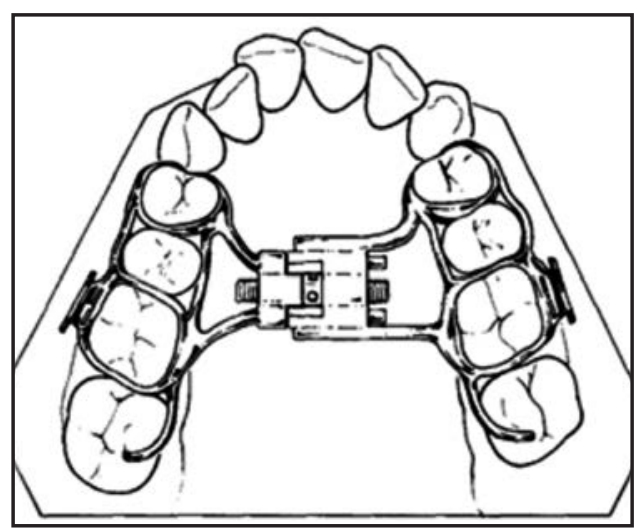

Figura 6. Expansión rápida maxilar. 
intervención, el índice de apnea-hipopnea bajó a $<1$ evento por hora en todos los casos, la rinometría se normalizó y se logró una expansión maxilar media de $4.32 \mathrm{~mm}$. Con este resultado postulan que la ERM es una opción útil en el tratamiento del SAOS en niños sin hiperplasia adenoamigdalina y con las alteraciones maxilares descritas.

En el caso de los niños con TRS secundario a hiperplasia adenoamigdalina y alteraciones del crecimiento craneofacial, ¿qué debe tratarse primero? Esta interrogante no está del todo clara y generalmente se tiende a realizar el tratamiento que ofrece el especialista a quien consulta primero el paciente. Guilleminault y $\operatorname{cols}^{16}$, realizaron un estudio randomizado con 31 pacientes diagnosticados de SAOS por hiperplasia adenoamigdalina con maloclusiones con indicación de ERM. Se dividieron en 2 grupos, el primer grupo fue sometido a adenoamigdalectomía seguido de ERM mientras que el segundo grupo fue tratado primero con ERM y luego adenoamigdalectomía. Todos los niños después de recibir el primer tratamiento presentaron una mejoría de los síntomas, pero ninguno se normalizó. Tras recibir el segundo tratamiento se logró la resolución definitiva de los síntomas y normalización de la polisomnografía, presentado diferencias estadísticamente significativas respecto al basal, pero sin diferencias entre ambos grupos, concluyendo que no hay diferencia en el orden de los tratamientos ya que la resolución definitiva se logra complementando ambos tratamientos.

Güray y $\operatorname{cols}^{17}$, estudiaron los efectos de la adenoidectomía en el crecimiento dentofacial. Para esto evaluaron a 26 niños con diagnóstico de obstrucción nasal secundaria a hiperplasia adenoidea con indicación de adenoidectomía. 13 de estos pacientes fueron sometidos a adenoidectomía y 13 solamente fueron observados constituyendo el grupo control. Se realizaron medidas cefalométricas al inicio del estudio y 6 años después, planimetría digital para determinar el área respiratoria y nasofaríngea y la resistencia nasal, y moldes de la dentadura para medir el ancho y el largo del arco maxilar, la altura del paladar, el ancho y largo del arco mandibular. En este estudio se observó una disminución de la resistencia nasal y alteraciones en la mordida en el grupo de tratamiento en comparación al grupo control, pero no hubo diferencias estadísticamente significativas en el crecimiento dentofacial. Por lo tanto se concluyó que la adenoidectomía por sí sola juega un rol en la restauración de la respiración nasal pero no en la regularización del desarrollo craneofacial. El promedio de edad de los niños era de 9,1 años \pm 2 años. Por lo que probablemente la intervención fue muy tardía ya que a los 9 años de edad ya ha terminado el crecimiento horizontal de maxilar y mandíbula ${ }^{18}$.

\section{CONCLUSIONES}

Los trastornos respiratorios del sueño son frecuentes en la edad pediátrica. Recordar que tanto el roncador primario como el síndrome de apnea obstructiva del sueño pueden cursar con respiración bucal la cual desencadena una serie de alteraciones en el crecimiento craneofacial del niño, destacando la presencia de paladar ojival que conlleva a un piso nasal más alto con fosas nasales más angostas y bajas, por lo que ante una mínima desviación septal y/o crecimiento de cornetes, se verá afectada con mayor facilidad la permeabilidad nasal. Una mandíbula pequeña que se relaciona con una lengua proporcionalmente de mayor tamaño y por ende, un valor mayor en la escala de Mallampati, nos puede afectar a la columna aérea oro e hipofaringea.

Si bien la adenoamigdalectomia es el tratamiento de elección en SAOS en el niño, siempre se deben valorar otras patologías asociadas como la obesidad, enfermedades neuromusculares y las malformaciones craneofaciales, las cuales se asocian a una mayor tasa de SAOS residual.

El tratamiento de la respiración bucal debe ser lo más precoz posible para disminuir el impacto en el niño. Es un factor significativo en la conformación del esqueleto maxilofacial durante la infancia, además se deben considerar los factores genéticos asociados. La intervención además de ser precoz, debe ser en conjunto entre el otorrinolaringólogo y el ortodoncista ortopedista, que sea capaz de modular el crecimiento maxilomandibular y no esperar hasta que éste se haya completado, y en esa tardía intervención, realizar acciones orientadas principalmente a corregir solo la oclusión.

Finalmente, la poligrafía del sueño ambulatoria nos aporta información suficiente para evaluar los diferentes trastornos respiratorios del sueño y poder confirmar su diagnóstico y respuesta a tratamiento de una forma más objetiva. 


\section{BIBLIOGRAFÍA}

1. Zenteno D, Salinas $P$, Vera R, Brockmann P, Prado F. Enfoque pediátrico para el estudio de los trastornos respiratorios del sueño. Rev Chil Pediatr 2010; 81 (5): 445-55.

2. Lı A, Au C, So H, Lau J, NG P, Wing Y. Prevalence and Risk Factors of Habitual Snoring in Primary School Children. Chest 2010; 138(3): 519-27.

3. Marcus Cl, Brooks LJ, Draper Ka, Gozal D, Halbower AC, Jones J, Schechter MS, Sheldon SH, Spruyt K, Ward SD, Lehmann C, Shiffman RN, American Academy of Pediatrics. Diagnosis and management of childhood obstructive sleep apnea syndrome. Pediatrics 2012; 130(3): 57684.

4. Brockmann P, Bertrand P, Pardo T, Cerda J, Reyes B, HoLgRen N. Prevalence of habitual snoring and associated neurocognitive consequences among Chilean school aged children. International Journal of Pediatric Otorhinolaryngology 76 (2012) 1327-31.

5. Brockmann P, Urschitz M, Schlaud M, Poets $C$. Primary snoring in school children: Prevalence and neurocognitive impairments.

6. Steiner C. Cephalometrics for you and me. Am J Orthod 1953; 39: 729-55.

7. FACAL A. La dirección del crecimiento de la cara como objetivo ortopédico del tratamiento. Control clínico cefalométrico. Rev Esp Ortod 2005; 35: 11-46.

8. Harvold E, Tomer B, Vargervik K, Chierici G. Primate experiments on oral respiration. American Journal of Orthodontics 1981; 79: 359-72.

9. KІкUСНI M. Orthodontic treatment in children to prevent sleep-disordered breathing in adulthood.
Sleep Breath 2005; 9: 146-58.

10. Praud JP, Dorion D. Obstructive Sleep Disordered breathing in Children: Beyond Adenotonsillectomy. Pediatric Pulmonology 2008; 43: 837-43.

11. Tasker C, Crosby JH, Stradling JR. Evidence for persistence of upper airway narrowing during sleep, 12 years after adenotonsillectomy. Arch Dis Child 2002; 86: 34-7.

12. Zettergren-WiJk L, Linder-Aronson S, Nordlander $B$, Agren K. Longitudinal effect on facial growth after tonsillectomy in children with obstructive sleep apnea. World J Orthod 2002; 3: 67-72.

13. Kım JH, Guilleminault C. The nasomaxillary complex, the mandible, and sleep-disordered breathing. Sleep Breath 2011; 15(2): 185-93.

14. Mata J, Zambrano F, Quirós 0, Maza P, Jurisic A, Alcedo C, Fuenmayor D, Ortiz M. Expansión rápida de maxilar en maloclusiones transversales: revisión bibliográfica. Revista latinoamericana de ortodoncia y odontopediatría edición electrónica octubre 2009. Disponible en www.ortodoncia. ws. Consultado el 19/11/2015.

15. Pirelli P, Saponara M, Guilleminault C. Rapid maxillary expansión in children with obstructive sleep apnea síndrome. Sleep 2004; 27(4): 761-6.

16. Guilleminault $C$, Monteyrol PJ, Huynh N, Pirelli P, Quo S, Lı K. Adeno-tonsillectomy and rapid maxillary distraction in pre-pubertal children, a pilot study. Sleep Breath 2011; 15: 173-7.

17. Güray E, Karaman A. Effects of adenoidectomy on dentofacial structures: A 6-year longitudinal study. World J Orthod 2002; 3: 73-81.

18. Profitt W, Fields H, Sarver D. Contemporary Orthodontics. Mosby, Fifth edition (2012). Chapter 2 Concepts of growth and development: 26-72. 